\title{
SHORT REPORT \\ Impact on the medical decision-making process of multiplex PCR assay for respiratory pathogens
}

\author{
A. GUILLON ${ }^{1}$, S. AYMERIC ${ }^{2}$, C. GAUDY-GRAFFIN ${ }^{3}$, J. SONKE$^{2}$,

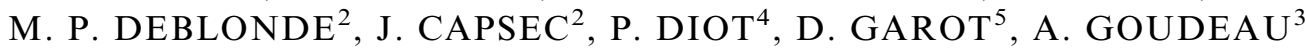 \\ AND L. GRAMMATICO-GUILLON ${ }^{6}$ \\ ${ }^{1}$ Service de Réanimation Polyvalente, CHRU de Tours, Université François Rabelais, CEPR, U1100, Tours, France \\ ${ }^{2}$ Laboratoire de Santé Publique, CHRU de Tours, Université François Rabelais, EE Education Ethique Santé, \\ Tours, France \\ ${ }^{3}$ Service de Microbiologie, CHRU Tours, Université François Rabelais, U966, Tours, France \\ ${ }_{5}^{4}$ Service de Pneumologie, CHRU de Tours, Université François Rabelais, CEPR, U1100, Tours, France \\ ${ }^{5}$ Service de Réanimation Polyvalente, CHRU de Tours, Tours, France \\ ${ }^{6}$ Laboratoire de Santé Publique, CHRU de Tours, Unité Régionale d'Épidémiologie Hospitalière, U966, Tours, \\ France
}

Received 5 December 2016; Final revision 31 March 2017; Accepted 20 April 2017; first published online 18 May 2017

\section{SUMMARY}

The objective of this study was to determine how clinicians make use of the modern multiplex PCR assays (MPAs) to manage patients hospitalized for community-acquired pneumonia (CAP). We studied the use of MPAs in 1648 patients hospitalized for CAP over a 3-year period at the moment of the setup of the new PCR assay. We observed that the use of MPAs for the identification of multiple respiratory pathogens marks a radical change in the investigation of CAP etiology. Surprisingly, the contribution of MPAs to the medical decision-making process varies drastically according to the units of care.

Key words: Community-acquired pneumonia, medical decision-making, multiplex PCR assay.

Community-acquired pneumonia (CAP) is a major global healthcare burden associated with significant morbidity, mortality and cost [1]. Identifying the etiology of CAP is the first challenge in its management. The broad spectrum of pathogens involved in CAP includes leading disease-causing agents, such as Streptococcus pneumoniae, several fastidious bacteria and viruses. Treatment decisions are nowadays essentially based on epidemiological findings, but more personalized approaches are needed [2-4]. To this end, combined multimolecular-diagnosis methods have

\footnotetext{
* Author for correspondence: Dr L. Grammatico-Guillon, MD PhD, Teaching Hospital of Tours, Regional Unit of Epidemiology, 2 BD Tonnellé 37044 tours cedex 9, France. (Email: Leslie.guillon@univ-tours.fr)
}

been developed for the identification of a large panel of bacterial and viral agents in single clinical samples. Multiplex PCR assays (MPAs) for the rapid identification of respiratory pathogens constituted a particular breakthrough [5]. However, clinicians are now faced with a new challenge: information overload. Increase in the sensitivity of molecular methods for the detection of viruses in nasopharyngeal secretions has made their clinical interpretation more difficult. How can we be sure that a microorganism detected in the sample is the pathogen responsible for disease [6]? Moreover, the detection of influenza, adenovirus, or respiratory syncytial viruses (generally assumed to be disease-causing agents) does not exclude the possibility of coinfection or superinfection with bacteria or other viruses. These new tests may ultimately modify 
clinician behavior but it remains unclear how best to combine MPA results with clinical assessment and other types of information to achieve an accurate diagnosis and provide the most appropriate treatment. The objective of this study was to determine the impact of MPA on the medical decision-making process to manage patients hospitalized for CAP. To this aim, we assessed three questions: Did the clinicians use the MPAs? Were the results provided by the MPAs useful for the etiological diagnosis? Did it lead to treatment modification?

We conducted a retrospective cross-sectional study based on hospital discharge databases (HDDs), covering a 3-year period. We selected patients over 18 years old who were hospitalized in the intensive care unit (ICU) or the Pulmonology Department of a French Teaching Hospital during the three winter periods of 2011-2014 (defined from week 38 of year $N-1$ to week 18 of year $N$ ). Cases of CAP were extracted from the HDD with an algorithm based on specific diagnosis codes from the International Classification of Diseases, 10th revision (ICD-10) for pneumonia (ICD-10 codes: J10-J18), taking into account the type, number and position of these codes in the hospital discharge report. We next validated the ICD-10 case definition reviewing a sample of medical charts as the gold standard and calculated the positive and negative predictive values of this algorithm of case selection. We reviewed 570 medical records: 287 patients with CAP recorded in the HDD, and 283 controls presenting respiratory diseases recorded in the HDD but without CAP (i.e. exacerbation of chronic obstructive pulmonary disease without pneumonia). Clinicians performed case validation blindly according to the patient's medical chart. A positive predictive value of $83 \%$ and a negative predictive value of $95 \%$ were obtained. Giving the acceptable accuracy and precision of our algorithm, the following data were extracted from the HDD: patient characteristics, use of assisted ventilation, length of stay, occurrence of death. MPA data were extracted from the database of the virology laboratory and were used to describe the utilization of the MPA over time and between services.

Two multiplex assays were used during the study period. RespiFinder ${ }^{\mathbb{R}}$ SMART 22 (PathoFinder) was used during the first 2 years (2011-2012 and 20122013). This MPA can be used for the simultaneous qualitative detection of 18 respiratory viruses influenza A, B, and A-H1N1pdm2009 virus, respiratory syncytial viruses $\mathrm{A}$ and $\mathrm{B}$, parainfluenza viruses
1-4, coronaviruses OC43, 229E, NL63, and HKU1, rhinovirus/enterovirus, human metapneumovirus, adenovirus, human bocavirus - and four bacteria (Legionella pneumophila, Chlamydophila pneumoniae, Mycoplasma pneumoniae, and Bordetella pertussis). Anyplex II RV16 and RB5 assays (Seegene) were used in 2013-2014. These assays were able to detect Bordetella parapertussis in addition to the pathogens listed above. MPA testing was performed once per week during the first year of the study period, twice per week during the second winter and every working day in 2013-2014. We assessed the impact of PCR results on the etiological diagnosis and potential treatment modifications by focusing on the last winter period (2013-2014), during which the use of MPAs made it possible to obtain results in a clinically relevant timeframe. We retrospectively reviewed all medical charts including positive MPA results for the period 2013-2014. We considered the information provided by the MPA to have been useful for the clinician to define the etiological diagnosis if the microorganism identified was mentioned in the conclusion of the final medical report for the patient. We also used the recorded changes to antibiotic therapy (stop or simplification) due to positive MPA results as the criterion of MPA use for medical decision.

We studied 1648 patients diagnosed with CAP over the 3-year period. The median total length of stay was 6 days. In-hospital mortality was $8.9 \%$. Mechanical ventilation was required in $33-42 \%$ of patients hospitalized in the ICU (Fig. 1a). The use of MPAs progressively increased from 2011 to 2013, resulting in a higher frequency of testing, reducing the turnaround time from 68 to 8 hours (Fig. 1b). This innovative technology was used very differently in the two departments. Overall, $62 \%$ of MPA performed were positive. In the ICU, the number of subjects tested with MPAs increased steadily over the 3 years: $41.5 \%$ of patients having been tested the first year of MPA use and $70 \cdot 2 \% 3$ years later (Fig. 1c). By contrast, the clinicians of the pulmonology department rarely requested MPAs for their patients. Tests were carried out for only $7 \cdot 2 \%$ of pulmonary department patients in 2011, and this already low proportion decreased still further over time, with only $2 \cdot 9 \%$ tested in 2013 (Fig. 1c). Obviously, there are clear differences between the patients admitted to ICUs and pulmonology departments. However, the same method was used to collect nasopharyngeal secretions in both departments. Furthermore, the proportion of patients on mechanical ventilation and, therefore, potentially 
(a)

\begin{tabular}{|c|c|c|c|}
\hline & 2011-12 & 2012-13 & 2013-14 \\
\hline Patients (n) & 563 & 537 & 548 \\
\hline Mean age (+/-SD) & $68 \cdot 1(+/-17 \cdot 8)$ & $69.5(+/-16.4)$ & $68 \cdot 7(+/-17 \cdot 2)$ \\
\hline Range of age (years) & $18-99$ & $20-100$ & $19-105$ \\
\hline Sex ratio $(M / F)$ & 1.5 & 1.4 & 1.6 \\
\hline Mean comorbid conditions (+/-SD) ${ }^{\#}$ & $1.7(+/-1.4)$ & $1 \cdot 6(+/-1 \cdot 3)$ & $1.7(+/-1.4)$ \\
\hline \multirow{5}{*}{ Comorbidity frequency $(\%)$} & $19 \cdot 6$ & 21.8 & $21 \cdot 4$ \\
\hline & $30 \cdot 3$ & $29 \cdot 2$ & $28 \cdot 3$ \\
\hline & $23 \cdot 2$ & $25 \cdot 2$ & $26 \cdot 6$ \\
\hline & $15 \cdot 4$ & $14 \cdot 1$ & $12 \cdot 9$ \\
\hline & 11.5 & $9 \cdot 7$ & $10 \cdot 9$ \\
\hline \multirow{2}{*}{$\begin{array}{r}\text { Hospital stays }(n, \%) \quad \text { ICU } \\
\text { Pulmonary department }\end{array}$} & $270(45 \cdot 7)$ & $256(45 \cdot 1)$ & $205(35 \cdot 3)$ \\
\hline & $321(54 \cdot 3)$ & $312(54 \cdot 9)$ & $375(64 \cdot 7)$ \\
\hline Rehospitalizations ( $n, \%$ ) & $28(4 \cdot 7)$ & $31(5 \cdot 5)$ & $32(5.5)$ \\
\hline Assisted ventilation in ICU (\%) & 34 & 33 & 42 \\
\hline \multirow{2}{*}{$\begin{array}{l}\text { Length of stay Median (range) in days } \\
\text { Death }(n, \%)\end{array}$} & $7(1-120)$ & $7(1-167)$ & $6(1-160)$ \\
\hline & $64(11.4)$ & $43(7 \cdot 6)$ & $40(6.9)$ \\
\hline
\end{tabular}

(b)

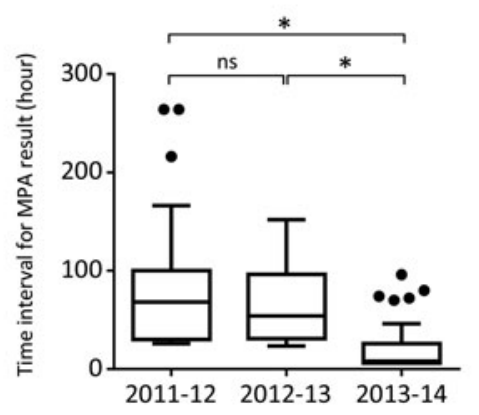

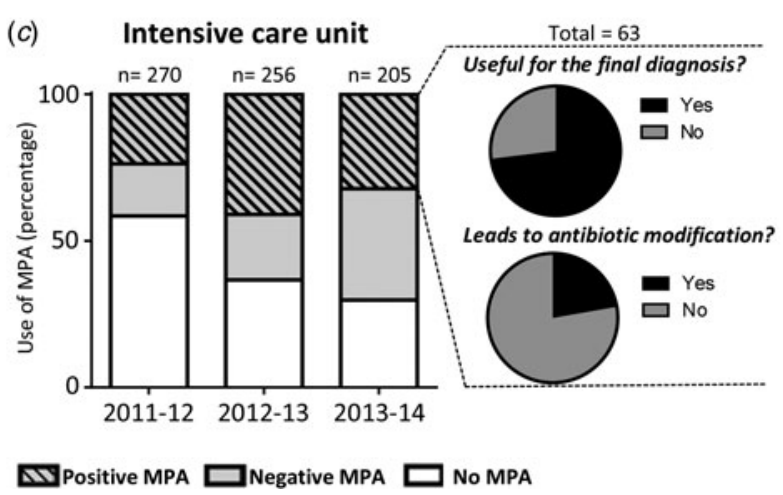

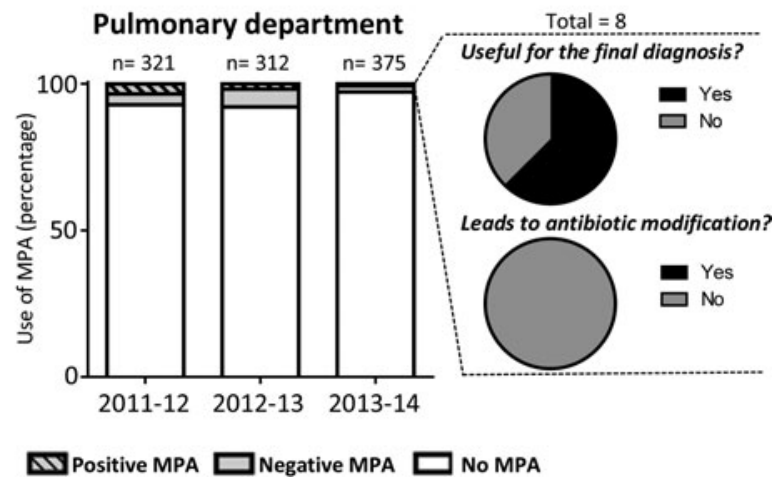

Fig. 1. Utility of MPAs for the medical management of patients diagnosed with community-acquired pneumonia (CAP). Patients hospitalized for CAP in the ICU or the pulmonary department between 2011 and 2014 were retrospectively analyzed. (a) The baseline characteristics of study participants are reported in the table. (b) The turnaround times for MPAs are represented as Tukey box-and-whisker plots, by year of analysis. Non-parametric Kruskal-Wallis and Dunn's multiple comparison tests were used to compare these quantitative variables. $(c)$ The percentages of MPAs yielding positive and negative results are reported. All medical charts reporting positive MPA results for the 2013-2014 period were studied, to address the following two questions: (i) 'Was the MPA result useful for the final etiological diagnosis?' We considered the answer to this question to be 'yes' if the microorganism identified by the MPA was mentioned in the conclusion of the final medical report for the patient; (ii) 'Did the MPA result lead to any change in antibiotic treatment?' We considered the answer to this question to be 'yes' if any change in antibiotic treatment due to the MPA result was noted in the patient's medical records. S.D., standard deviation; ICU, intensive care unit; MPA, multiplex PCR assay; \#, Comorbid conditions according to hospital discharge database codes linked to the Elixhauser and Charlson comorbidity indices; * $P<0 \cdot 05$.

sedated in the ICU could not account for the magnitude of the difference.

We evaluated the impact on the decision-making process of this increasing amount of microbiological information when the MPAs were performed daily (last winter period 2013-2014). A positive microbiological identification was obtained for $47.7 \%$ of the ICU patients tested. This result is consistent with previous findings $[7,8]$. This positive information seemed to be relevant for the clinical decision-making process as it was reported in the final diagnosis of $73 \%$ of patients. Ultimately, pathogen identification led to changes in antibiotic treatment in $22 \%$ of patients. Very few patients from the pulmonology department were tested, and the results obtained were, therefore, difficult to analyse. In this ward, MPA results were noted on the patient's medical chart to conclude on the etiology of CAP but had ultimately no impact on treatment decisions.

MPAs are a promising approach to improving diagnosis, but these improvements will entail a significant increase in cost. It is, therefore, important to evaluate continually their direct usefulness and impact, in terms of better patient care. The use of MPAs for the identification of multiple respiratory pathogens marks a radical change in the investigation of CAP etiology. In the ICU, MPAs were widely used and made a key 
contribution to the medical decision-making process. By contrast, MPAs were used for only a few selected patients in the pulmonology department and the results obtained had no impact on treatment decisions.

Further studies are required to assess the costeffectiveness of MPAs and their contribution to overall patient outcome.

\section{REFERENCES}

1. Grammatico-Guillon L, et al. Study of hospitalizations for pneumococcal pneumoniae in centre region, 2004-2008. Revue d'Epidemiol Sante Publique 2012; 60: 1-8.

2. Wunderink RG, Waterer GW. Clinical practice. Community-acquired pneumonia. New England Journal Medicine 2014; 370: 543-551.

3. Vincent J-L, et al. Rapid diagnosis of infection in the critically Ill, a multicenter study of molecular detection in bloodstream infections, pneumonia, and sterile site infections. Critical Care Medicine 2015; 43: 2283-2291.

4. Gadsby NJ, et al. Comprehensive molecular testing for respiratory pathogens in community-acquired pneumonia. Clinical Infectious Diseases 2016; 62: 817-823.

5. Subramony A, et al. Impact of multiplex polymerase chain reaction testing for respiratory pathogens on healthcare resource utilization for pediatric inpatients. Journal of Pediatrics 2016; 173: 196-201.

6. Contentin L, et al. Acute respiratory distress syndrome secondary to human metapneumovirus infection in a young healthy adult. Intensive Care Medicine 2013; 39: 533-534.

7. Schnepf $\mathbf{N}$, et al. High burden of non-influenza viruses in influenza-like illness in the early weeks of H1N1v epidemic in France. PLoS ONE 2011; 6: e23514.

8. Burk M, et al. Viral infection in community-acquired pneumonia: a systematic review and meta-analysis. European Respiratory Revue 2016; 25: 178-188. 